\title{
MicroRNA-29a inhibits cell migration and invasion by targeting Roundabout 1 in breast cancer cells
}

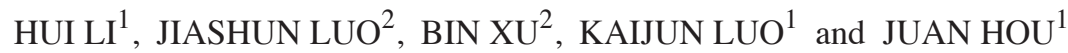 \\ ${ }^{1}$ Department of Microbiology and Immunology; ${ }^{2}$ Institute of Medical Research, \\ Medical School of Jishou University, Jishou, Hunan 416000, P.R. China
}

Received July 29, 2014; Accepted April 16, 2015

DOI: $10.3892 / \mathrm{mmr} .2015 .3749$

\begin{abstract}
MicroRNAs (miRs) are a group of small non-coding RNAs, which influence the development and progression of cancer by mediating the protein expression levels of tumor suppressors or oncogenes. miR-29a has been reported to be involved in the pathogenesis of breast cancer. However, its precise functions, and the mechanisms underlying these effects, remain to be elucidated. The present study demonstrated that miR-29a was markedly downregulated in breast cancer tissues and cell lines. The protein expression of Roundabout 1 (Robo1) was negatively regulated by miR-29a in MCF-7 breast cancer cells. Luciferase reporter assays identified Robol as a direct target of miR-29a in MCF-7 cells. Additionally, upregulation of miR-29a inhibited MCF-7 cell migration and invasion, which was attenuated by the restoration of the expression of Robol. Finally, it was shown that the expression of Robol mRNA and protein was increased in breast cancer tissues and cell lines. Therefore, it was hypothesized that miR-29a inhibits cell migration and invasion in breast cancer cells, at least in part, by directly targeting Robo1. In conclusion, the present study highlights the importance of miR-29a and Robol in the progression of breast cancer.
\end{abstract}

\section{Introduction}

Breast cancer is a common malignant tumor in females, accounting for a high proportion of cancer-associated mortality (1). However, the molecular mechanisms underlying breast cancer remain to be elucidated. The identification of potential molecular targets for the treatment of breast cancer appears promising (2).

MicroRNAs (miRNAs) are a class of short ( 20 nt) non-coding RNAs, which bind to the 3'-untranslated region

Correspondence to: Dr Hui Li, Department of Microbiology and Immunology, Medical School of Jishou University, 120 Renminnan Road, Jishou, Hunan 416000, P.R. China

E-mail: jishoulihui123@163.com

Key words: breast cancer, microRNA-29a, Roundabout 1, migration, invasion
(UTR) of their target mRNAs, and exert a suppressive effect on protein translation or induce degradation of the mRNA (3). By negatively regulating the protein expression of their targets, miRNAs influence various biological processes, including cell survival, proliferation, differentiation and motility (4-6). The deregulation of miRNAs has been demonstrated to be associated with the development and progression of multiple types of human malignancy, including breast cancer, indicating that these molecules act as tumor suppressors or oncogenes $(7,8)$. Among the miRNAs investigated, miR-29a has been shown to act as a tumor suppressor in several types of human cancer, including prostate cancer, colorectal cancer, oral squamous carcinoma and breast cancer (9-12). Wu et al reported that miR-29a exerted an inhibitory effect on breast cancer cell growth (9). However, the precise function of miR-29a in the regulation of cell migration and invasion in breast cancer cells, and the mechanisms underlying its effects on these processes remain to be elucidated.

The secreted Slit glycoproteins and their Roundabout (Robo) receptors were originally identified as important axon guidance molecules $(13,14)$. Robol is a member of Robo family, which is expressed in multiple cell types and is important in the regulation of various biological processes, including cell proliferation, differentiation and migration (15-17). A previous study demonstrated the involvement of Robol in cancer cell migration and invasion (18). In addition, a bioinformatic analysis suggested that Robo1 is a putative target of miR-29a (19). However, the underlying mechanisms by which Robol mediates breast cancer remain to be elucidated.

The present study primarily aimed to investigate the effects of miR-29a and Robol on the regulation of cell migration and invasion in breast cancer cells. The mechanisms underlying these effects were also examined.

\section{Materials and methods}

Tissue specimen collection. The present study was approved by the Ethical Committee of Jishou University (Jishou, China). Breast cancer tissues and matched adjacent normal tissues $(n=18)$ were obtained from the Department of General Surgery, People's Hospital of Xiangxi Autonomous Prefecture, Jishou University (Jishou, China). Written informed consent was obtained from patients with breast cancer. The tissue samples were frozen in liquid nitrogen following surgery, until use. 
Cell culture. T47D, MCF-7, BCAP-37 and MDA-MB-435S human breast cancer cell lines, and the MCF-10A normal breast epithelial cell line, were purchased from ScienCell (San Diego, CA, USA). All cells were cultured in Dulbecco's modified Eagle's medium (DMEM; Life Technologies, Carlsbad, CA, USA), supplemented with $10 \%$ fetal bovine serum (FBS; Life Technologies) at $37^{\circ} \mathrm{C}$ with $5 \% \mathrm{CO}_{2}$.

Reverse transcription-quantitative polymerase chain reaction $(R T-q P C R)$. Total RNA was isolated using TRIzol reagent (Invitrogen Life Technologies, Carlsbad, CA, USA), according to the manufacturer's instructions. For miRNA detection, total RNA was reverse transcribed using the miScript Reverse Transcription kit (Qiagen, Valencia, CA, USA), according to the manufacturer's instructions. RT-qPCR of the miRNA was then performed using the miScript SYBR Green PCR kit (Qiagen) on an ABI7500 PCR machine (Invitrogen Life Technologies). RT-qPCR was conducted at $95^{\circ} \mathrm{C}$ for $15 \mathrm{~min}$, followed by 40 cycles of $94^{\circ} \mathrm{C}$ for $15 \mathrm{sec}, 55^{\circ} \mathrm{C}$ for $30 \mathrm{sec}$ and $70^{\circ} \mathrm{C}$ for $30 \mathrm{sec}$. The relative expression of miRNA was normalized against that of U6. For mRNA detection, the total RNA was reverse transcribed into cDNA using the RevertAid First-Strand cDNA Synthesis kit (Fermentas, Carlsbad, CA, USA), according to the manufacturer's instructions. RT-qPCR was subsequently performed using iQTM SYBR Green Supermix (Bio-Rad, Hercules, CA, USA) by denaturation at $95^{\circ} \mathrm{C}$ for $15 \mathrm{~min}$, followed by 40 cycles of $94^{\circ} \mathrm{C}$ for $15 \mathrm{sec}, 55^{\circ} \mathrm{C}$ for $30 \mathrm{sec}$ and $70^{\circ} \mathrm{C}$ for $30 \mathrm{sec}$. The specific primers used were as follows: Forward: 5'-GGCGGTGAAGGAGATGAAC-3' and reverse: 5'-TGATGAGGAAATCCACGATAGAG-3' for Robol and forward: 5'-ACAACTTTGGTATCGTGGAAGG-3' and reverse: 5'-GCCATCACGCCACAGTTTC-3' for GAPDH. The relative mRNA expression of Robol was normalized against that of GAPDH.

Western blot assay. Cells were lysed in radioimmunoprecipitation buffer (Sigma-Aldrich, St. Louis, MO, USA). The total protein was isolated and the concentration was determined using the bicinchoninic acid protein assay kit (Santa Cruz Biotechnology, Inc., Santa Cruz, CA, USA). For western blot analysis, equal quantities of protein were boiled and separated using 10\% SDS-PAGE gels (Sigma-Aldrich), and blotted onto polyvinylidene difluoride (PVDF) membrane (Life Technologies), which was blocked with 5\% non-fat dried milk in phosphate-buffered saline (PBS) for $1.5 \mathrm{~h}$ at room temperature. The PVDF membrane was subsequently incubated with rabbit polyclonal anti-Robo1 (1:50; cat. no. ab7279) or rabbit polyclonal anti-GAPDH (1:50; cat. no. ab181602) antibodies (Abcam, Cambridge, MA, USA) at room temperature for $3 \mathrm{~h}$. The membranes were washed with PBS, and were then incubated with goat anti-rabbit immunoglobulin G H\&L secondary antibody (1:5,000; cat. no. ab175773; Abcam) for $40 \mathrm{~min}$ at room temperature. Chemiluminescent detection was performed using an enhanced chemiluminescence kit (Pierce Biotechnology, Inc., Rockford, IL, USA).

Transfection. Transfections were performed using lipofectamine 2000 (Invitrogen Life Technologies), according to the manufacturer's instructions. For miR-29a functional analysis, the cells were transfected with scrambled miRNA as a negative control (NC), miR-29a mimics or an miR-29a inhibitor (Invitrogen Life Technologies). For Robol functional analysis, the cells were transfected with Robol-specific small interfering RNA (siRNA) or Robol plasmid (Nlunbio, Changsha, China).

Luciferase reporter assay. A luciferase reporter assay was performed in MCF-7 human breast cancer cells. The wild-type (WT) 3'-UTR of Robol mRNA or the mutant type (MUT) 3'-UTR of Robo1 mRNA were inserted downstream of the luciferase reporter gene in the pMIR-REPORT vector (Life Technologies). Subsequently, cells were cotransfected with miR-29a mimics or scrambled miRNA; pMIR-REPORT vectors containing the WT or MUT Robol 3'-UTR; and pRL-SV40 (Promega Corporation, Sunnyvale, CA, USA), expressing Renilla luciferase. The cells were cultured for $48 \mathrm{~h}$ following transfection and the luciferase activities were measured using the Dual-Luciferase Reporter assay system (Promega Corporation).

Cell migration and invasion assay. Cell migration and invasion assays were performed using transwell chambers (BD Bioscience, Franklin Lakes, NJ, USA). Cell suspension, containing $5 \times 10^{5}$ cells $/ \mathrm{ml}$, was prepared in serum-free media. For cell migration assays, $300 \mu \mathrm{l}$ cell suspension was added into the upper chamber of the transwells. For cell invasion assays, $300 \mu \mathrm{l}$ cells were added into the upper chamber of transwells that were pre-coated with matrigel (BD Bioscience). DMEM (500 $\mu \mathrm{l})$, containing $10 \% \mathrm{FBS}$ as a chemo-attractant, was added into the lower chamber of the transwell. The cells were incubated for $24 \mathrm{~h}$ and cells, which failed to migrate or invade through the pores were carefully removed using a cotton-tipped swab. The filters were fixed in $90 \%$ alcohol and stained using crystal violet (Sigma-Aldrich). Cell numbers were determined in five randomly-selected fields under an inverted microscope (TS100; Nikon Corporatino, Tokyo, Japan).

Statistical analysis. The data are expressed as the mean \pm standard deviation of three independent experiments. SPSS 17.0 statistical software (SPSS, Inc., Chicago, IL, USA) was used to analyze the differences between the groups using one-way analysis of variance. $\mathrm{P}<0.05$ was considered to indicate a statistically significant difference.

\section{Results}

miR-29a is downregulated in breast cancer tissues and cells. The expression of miR-29a was determined using RT-qPCR in breast cancer tissues and matched normal adjacent tissues. As shown in Fig. 1A, the expression of miR-29a in breast cancer tissues was markedly reduced compared with that in the normal adjacent tissues. The expression of miR-29a was determined in four breast cancer cell lines, including T47D, MCF-7, BCAP-37 and MDA-MB-435S, and in one normal breast epithelial cell line, MCF-10A. As shown in Fig. 1B, miR-29a was also downregulated in breast cancer cell lines compared with the MCF-10A cell line. These findings suggested that miR-29a is involved in breast cancer progression. MCF-7 cells exhibited the greatest downregulation in the expression of miR-29a (Fig. 1B) and were therefore used for subsequent analysis. 
A

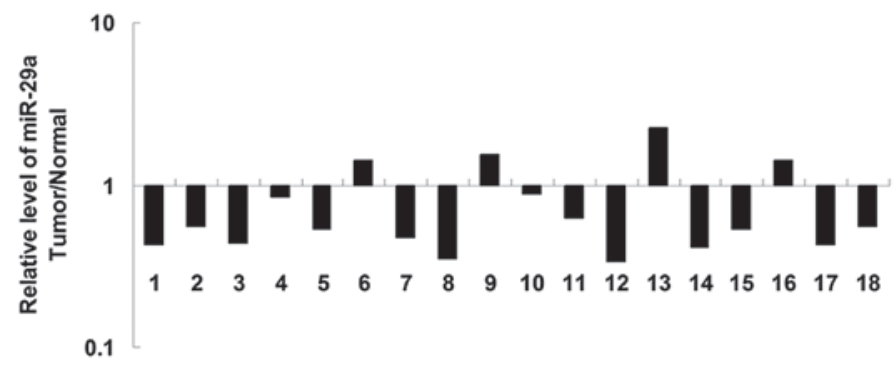

B

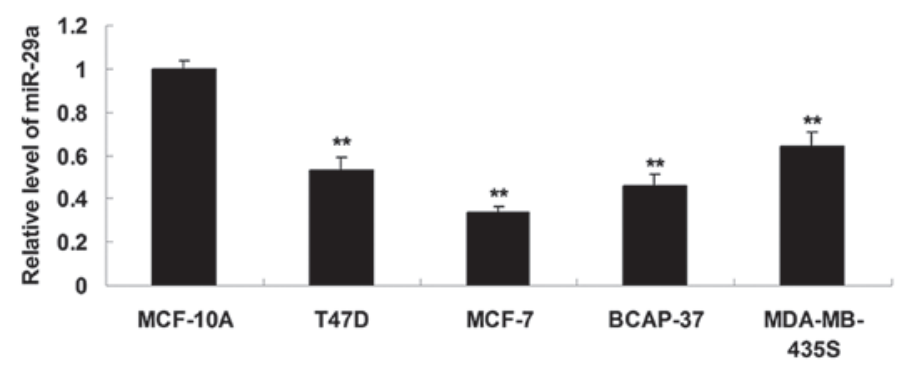

Figure 1. (A) RT-qPCR was performed to examine the expression of miR-29a in eighteen breast cancer tissues relative to that of matched normal adjacent tissues. (B) RT-qPCR was performed to examine the expression of miR-29a in the T47D, MCF-7, BCAP-37 and MDA-MB-435S human breast cancer cell lines relative to that in the MCF-10A normal breast epithelial cell line ( ${ }^{* *} \mathrm{P}<0.01$, vs. MCF-10A). RT-qPCR, reverse transcription-quantitative polymerase chain reaction; miR, microRNA.

A

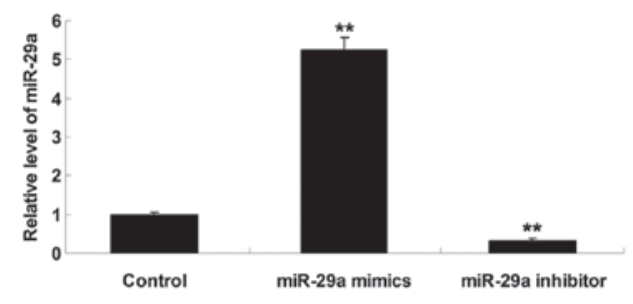

C

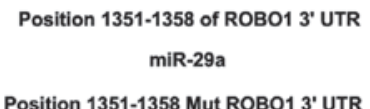

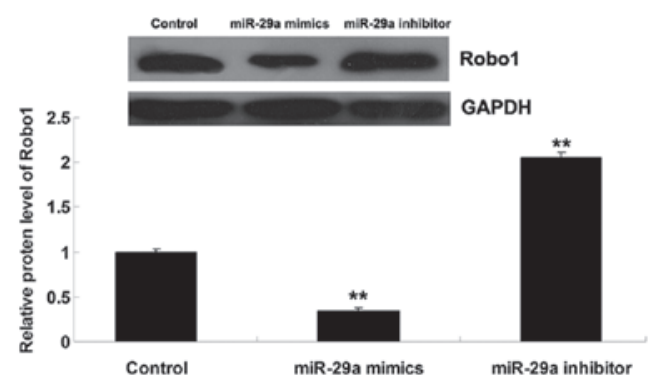

D

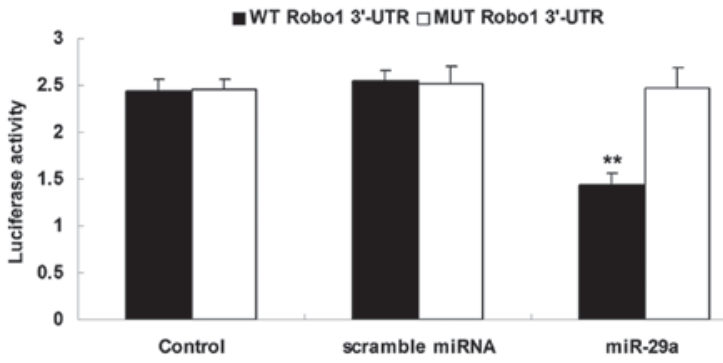

Figure 2. (A) Reverse transcription-quantitative polymerase chain reaction was performed to determine the expression of miR-29a in MCF-7 cells transfected with miR-29a mimics and miR-29a inhibitor, relative to that of the control group (MCF-7 cells without any transfection). (B) Western blotting was performed to assess the protein expression level of Robol in MCF-7 cells transfected with miR-29a mimics and miR-29a inhibitor. The control comprised MCF-7 cells without any transfection. (C) Putative binding sequences of miR-29a in the WT or MUT 3'-UTR in Robo1 mRNA. (D) Cotransfection of MCF-7 cells with miR-29a and WT Robol 3'-UTR caused a reduction in luciferase activity. However, cotransfection of MCF-7 cells with MUT Robol 3'-UTR and miR-29a mimics resulted in no difference in luciferase activity compared with the control group. The control comprised cells cotransfected with blank vector and WT or MUT Robol 3'-UTR, respectively. ${ }^{* * *} \mathrm{P}<0.01$, vs. control in each graph. miR, microRNA; Robo1, Roundabout 1; UTR, untranslated region; WT, wild-type; MUT, mutant.

miR-29a negatively regulates the protein expression of its target, Robol, in MCF-7 cells. The effect of miR-29a on the regulation of the expression of Robol was assessed in MCF-7 breast cancer cells. Following transfection with miR-29a mimics and an miR-29a inhibitor, the expression of miR-29a in MCF-7 cells was measured. As shown in Fig. 2A, transfection with miR-29a mimics caused an increase in the expression of miR-29a, while transfection with an miR-29a inhibitor led to downregulation of miR-29a, indicating that the transfection was successful. Subsequently, western blot analysis was performed to determine the protein expression levels of Robol in each group. As shown in Fig. 2B, upregulation of miR-29a led to a reduction in the expression of the Robo1 protein, whereas downregulation of miR-29a resulted in an increase in the expression of the Robol protein, indicating that the expression of Robol is negatively regulated by miR-29a 
A
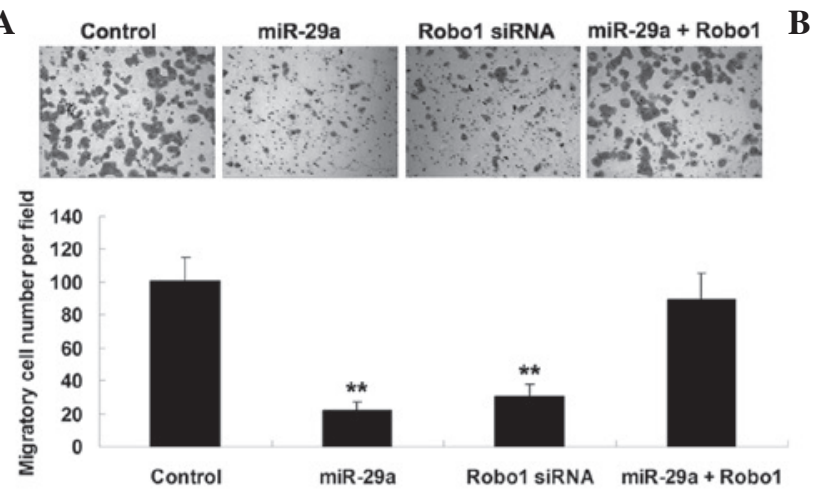
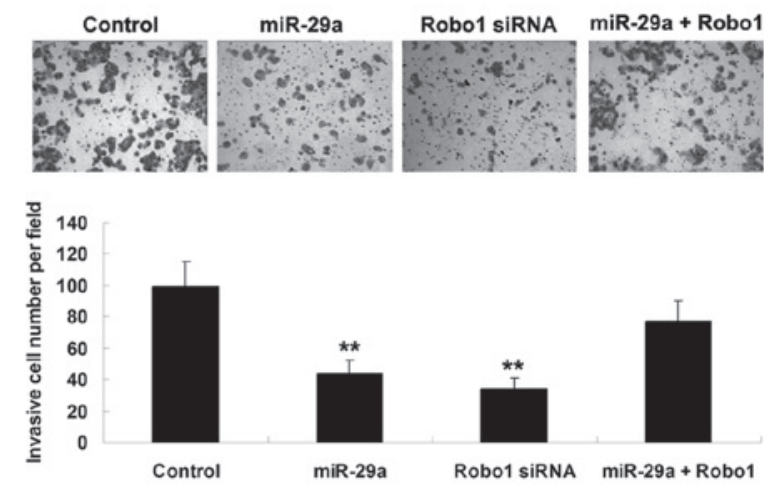

C

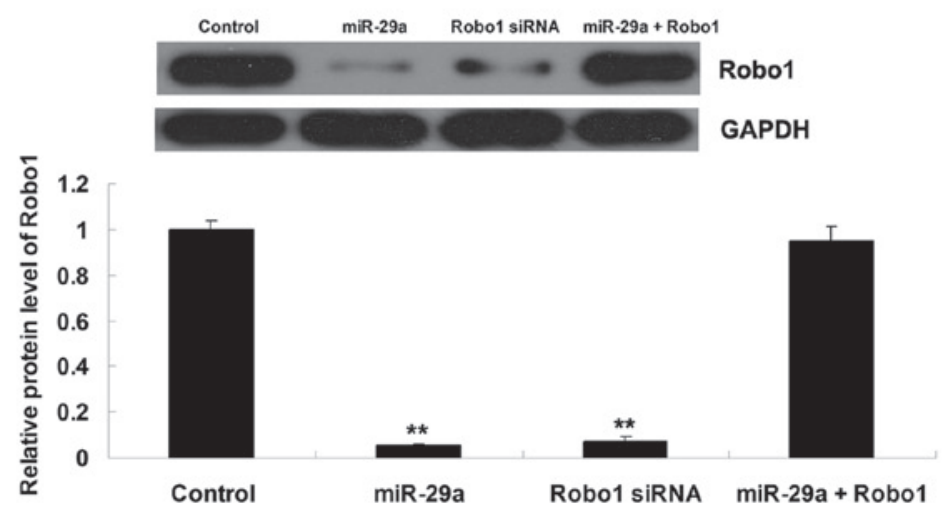

Figure 3. (A) A cell migration assay was performed in MCF-7 cells transfected with miR-29a mimics or Robo1-specific siRNA, or cotransfected with miR-29a mimics and Robol plasmid. The control comprised MCF-7 cells without any transfection. (B) A cell invasion assay was performed in MCF-7 cells transfected with miR-29a mimics or Robo1-specific siRNA, or cotransfected with miR-29a mimics and Robol plasmid. The control comprised MCF-7 cells without any transfection. (C) Western blotting was performed to determine the protein level of Robo1 in MCF-7 cells transfected with miR-29a mimics or Robol-specific siRNA, or cotransfected with miR-29a mimics and Robol plasmid. The control comprised MCF-7 cells without any transfection. * P $<0.01$, vs. control in each graph. miR, microRNA; siRNA, small interefering RNA; Robo1, Roundabout 1.

in MCF-7 breast cancer cells. A luciferase reporter assay was conducted in order to confirm whether miR-29a directly binds to the 3' UTR of Robo1 mRNA. As shown in Fig. 2C, a wild-type (WT-Robol) and a mutant (MUT-Robol) type of the Robol 3' UTR was generated. MCF-7 cells were cotransfected with the WT-Robol vector or the MUT-Robol vector, and the miR-29a mimics or a scrambled RNA. As demonstrated in Fig. 2D, the luciferase activity was significantly reduced compared with the control group, only in MCF-7 cells cotransfected with the WT-Robol vector and the miR-29a mimics. This repressive effect was abrogated in MCF-7 cells cotransfected with the MUT-Robol vector or the miR-29a mimics. These results suggested that miR-29a inhibits expression of the Robol protein via an interaction with the 3'-UTR of Robol.

miR-29a suppresses cell migration and invasion by targeting Robol in MCF-7 cells. The functions of miR-29a and Robo1, and their association in the regulation of cell migration and invasion were further assessed in MCF-7 breast cancer cells. MCF-7 cells were transfected with miR-29a mimics or Robol-specific siRNA, or cotransfected with miR-29a mimics and Robol plasmid. As shown in Fig. 3A and B, upregulation of miR-29a or downregulation of Robol significantly suppressed MCF-7 cell migration and invasion. However, the suppressive effects of miR-29a upregulation on MCF-7 cell migration and invasion were abrogated by overexpression of Robo1. Furthermore, western blotting was performed to determine the protein expression level of Robol in each group. As shown in Fig. 3C, the protein expression level of Robol was associated with the migratory and invasive capacities of MCF-7 cells in each group. These data suggested that miR-29a exerts a suppressive role in the regulation of cell migration and invasion, at least in part, through the direct inhibition of Robol expression in breast cancer cells.

Expression of Robol is increased in breast cancer tissues and cells. The level of Robol mRNA was subsequently determined in breast cancer tissues and matched normal adjacent tissues, using RT-qPCR. As shown in Fig. 4A, the mRNA level of Robol was significantly increased in breast cancer tissues compared with the normal adjacent tissues. In addition, the mRNA and protein expression levels of Robol were assessed in the T47D, MCF-7, BCAP-37 and MDA-MB-435S breast cancer cell lines, and in MCF-10A normal breast epithelial cells. As shown in Fig. 4B and C, Robol was upregulated in these four breast cancer cell lines compared with MCF-10A cells.

\section{Discussion}

Deregulation of miRNAs has been demonstrated to be involved in the progression of breast cancer (20). In the present study, the expression level of miR-29a was shown to be significantly reduced in breast cancer tissues and cell lines. Additionally, 
A

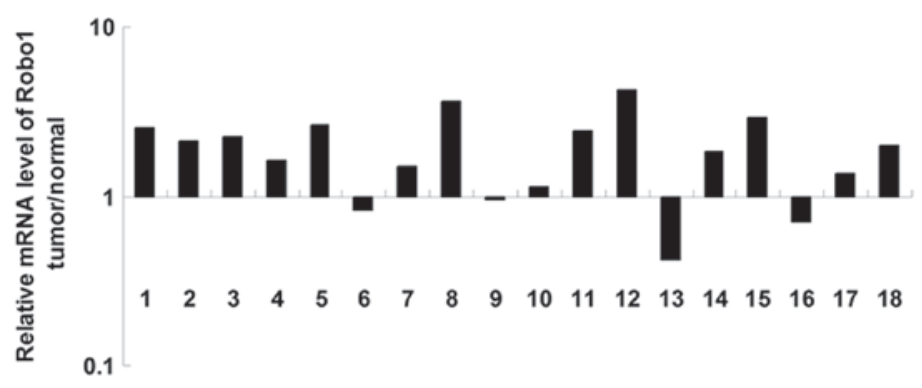

B

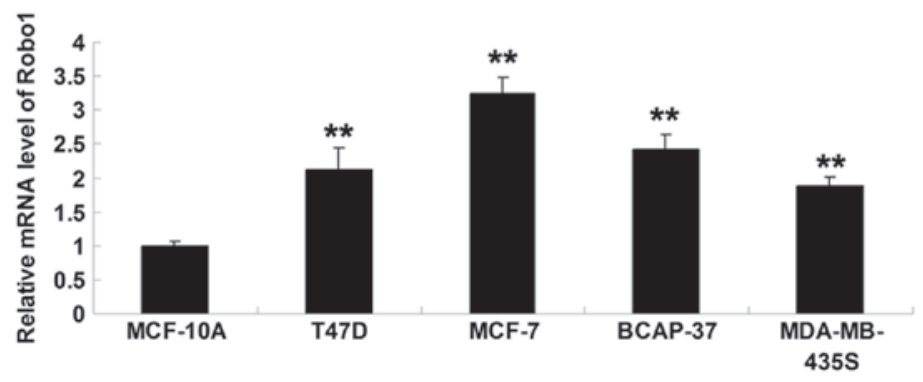

C

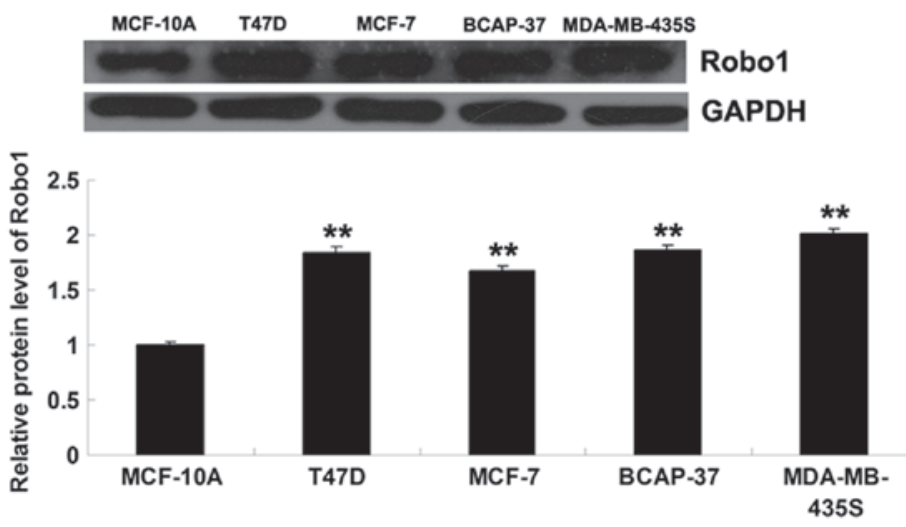

Figure 4. RT-qPCR was performed to determine the mRNA expression levels of Robol in (A) eighteen breast cancer tissues relative to matched normal adjacent tissues and (B) the T47D, MCF-7, BCAP-37 and MDA-MB-435S human breast cancer cell lines relative to that of the MCF-10A normal breast epithelial cell line. (C) Western blotting was performed to determine the protein expression levels of Robol in the T47D, MCF-7, BCAP-37 and MDA-MB-435S human breast cancer cell lines relative to that of the MCF-10A normal breast epithelial cell line. GAPDH was used as an internal loading control. ${ }^{* *} \mathrm{P}<0.01$, vs. MCF-10A. RT-qPCR, reverse transcription-quantitative polymerase chain reaction; Robo1, Roundabout 1.

Robol was identified as a novel target of miR-29a and its expression level was markedly upregulated in breast cancer tissues and cell lines. It was shown that the protein expression of Robo1 was negatively regulated by miR-29a and that miR-29a suppressed cell migration and invasion, at least in part, by directly targeting Robo1 in MCF-7 breast cancer cells.

Deregulation of miR-29a has been shown to contribute to multiple types of human malignancy. Yu et al (21) reported that downregulation of miR-29a contributes to cisplatin resistance in ovarian cancer cells. Zhao et al (22) demonstrated that miR-29a inhibited glioma tumor growth and invasion by targeting heat shock protein 47 . The present study demonstrated that miR-29a was significantly downregulated in breast cancer tissues compared with normal adjacent tissues. In addition, the expression level of miR-29a was also reduced in breast cancer cell lines compared with normal MCF-10A breast epithelial cells. miR-29a has been previously reported to be upregulated in serum, but downregulated in breast milk, in patients with breast cancer $(23,24)$. Furthermore, Wu et al (9) suggested an inhibitory role of miR-29a in breast cancer cells. The authors demonstrated that the overexpression of miR-29a significantly suppressed breast cancer cell proliferation and led to a higher percentage of cells in G0/G1 phase. In addition, the novel target, B-Myb, an important transcription factor associated with tumorigenesis, was identified (9). The present study revealed that miR-29a exerts an inhibitory function in the regulation of cell migration and invasion of MCF-7 breast cancer cells. Based on previous studies and the results of the present study, it is hypothesized that miR-29a acts as a key tumor suppressor in breast cancer.

Since a single miRNA may have different targets in different cancer cells, the present study aimed to identify a novel target that is involved in the progression of breast cancer. Robol was identified as a target of miR-29a in MCF-7 breast cancer cells. Robol was shown to be involved in tumorigenesis and the expression of Robol is known to be increased in several types of cancer (25). Alajez et al (26) demonstrated that Robol was upregulated in nasopharyngeal carcinoma, and its overexpression was significantly associated with a reduced level of overall and nodal relapse-free survival. In addition, Robol was also shown to be regulated by Src and Abl, and to promote tumor cell migration (27). Previously, Robol was 
shown to be involved in the regulation of cell migration and invasion in breast cancer cells (28). Yang et al (28) demonstrated that as one of the receptors for Slit, Robo1 is involved in miR-218-mediated inhibition of migration and invasion in breast cancer cells. The present study demonstrated a similar molecular mechanism, in which miR-29a inhibited cell migration and invasion by directly inhibiting the protein expression of Robol in breast cancer cells. Therefore, Robol is a common target for miR-29a and miR-218, each of which exert similar inhibitory effect on breast cancer cell migration and invasion. In addition, the oncogenic role of Robol in the regulation of cancer cell migration and invasion has been reported in other types of cancer. For instance, Tie et al (29) reported that Robol was negatively regulated by miR-218, and that this promoted the invasion and metastasis of gastric cancer (29).

In conclusion, the present study identified Robol as a direct target of miR-29a, and suggested that miR-29a exerts an inhibitory function in the regulation of cell migration and invasion, at least in part by suppressing the protein expression levels of Robol in breast cancer cells. Therefore, the results of the present study suggest that miR-29a and Robol may serve as potential targets for the treatment of metastatic breast cancer.

\section{References}

1. Murawa P, Murawa D, Adamczyk B and Polom K: Breast cancer: Actual methods of treatment and future trends. Rep Pract Oncol Radiother 19: 165-172, 2014.

2. Serpico D, Molino L and Di Cosimo S: microRNAs in breast cancer development and treatment. Cancer Treat Rev 40: 595-604, 2014.

3. Brower J, Clark PA, Lyon W and Kuo JS: MicroRNAs in cancer: Glioblastoma and glioblastoma cancer stem cells. Neurochem Int 77: 68-77, 2014.

4. Ishiguro H, Kimura M and Takeyama $\mathrm{H}$ : Role of microRNAs in gastric cancer. World J Gastroenterol 20: 5694-5699, 2014.

5. Bouyssou JM, Manier S, Huynh D, Issa S, Roccaro AM and Ghobrial IM: Regulation of microRNAs in cancer metastasis. Biochim Biophys Acta 1845: 255-265, 2014.

6. Kotaja N: MicroRNAs and spermatogenesis. Fertil Steril 101: $1552-1562,2014$.

7. Shah NR and Chen H: MicroRNAs in pathogenesis of breast cancer: Implications in diagnosis and treatment. World J Clin Oncol 5: 48-60, 2014.

8. Zhu J, Zheng Z, Wang J, et al: Different miRNA expression profiles between human breast cancer tumors and serum. Front Genet 5: 149, 2014.

9. Wu Z, Huang X, Zou Q and Guo Y: The inhibitory role of Mir-29 in growth of breast cancer cells. J Exp Clin Cancer Res 32: 98, 2013.

10. Nishikawa R, Goto Y, Kojima S, et al: Tumor-suppressive microRNA-29 s inhibit cancer cell migration and invasion via targeting LAMC1 in prostate cancer. Int J Oncol 45: 401-410, 2014.
11. Yang Y, Gu X, Zhou M, Xiang J and Chen Z: Serum microRNAs: A new diagnostic method for colorectal cancer. Biomed Rep 1: 495-498, 2013.

12. Lu L, Xue X, Lan J, et al: MicroRNA-29a upregulates MMP2 in oral squamous cell carcinoma to promote cancer invasion and anti-apoptosis. Biomed Pharmacother 68: 13-19, 2014.

13. Chaturvedi S and Robinson LA: Slit2-Robo signaling in inflammation and kidney injury. Pediatr Nephrol 30: 561-566, 2015.

14. Yang YH, Manning Fox JE, Zhang KL, MacDonald PE and Johnson JD: Intraislet SLIT-ROBO signaling is required for beta-cell survival and potentiates insulin secretion. Proc Natl Acad Sci USA 110: 16480-16485, 2013.

15. Yuen DA and Robinson LA: Slit2-Robo signaling: a novel regulator of vascular injury. Curr Opin Nephrol Hypertens 22: 445-451, 2013.

16. Cornide-Petronio ME and Barreiro-Iglesias A: Role of Slit and Robo proteins in the development of dopaminergic neurons. Dev Neurosci 35: 285-292, 2013.

17. Dickinson RE and Duncan WC: The SLIT-ROBO pathway: a regulator of cell function with implications for the reproductive system. Reproduction 139: 697-704, 2010.

18. Ballard MS and Hinck L: A roundabout way to cancer. Adv Cancer Res 114: 187-235, 2012.

19. Lewis BP, Burge CB and Bartel DP: Conserved seed pairing, often flanked by adenosines, indicates that thousands of human genes are microRNA targets. Cell 120: 15-20, 2005.

20. Zhong L, Zhu K, Jin N, et al: A systematic analysis of miRNA-mRNA paired variations reveals widespread miRNA misregulation in breast cancer. Biomed Res Int 2014: 291280, 2014.

21. Yu PN, Yan MD, Lai HC, et al: Downregulation of miR-29 contributes to cisplatin resistance of ovarian cancer cells. Int J Cancer 134: 542-551, 2014.

22. Zhao D, Jiang X, Yao C, et al: Heat shock protein 47 regulated by miR-29a to enhance glioma tumor growth and invasion. J Neurooncol 118: 39-47, 2014.

23. Wu Q, Wang C, Lu Z, Guo L and Ge Q: Analysis of serum genome-wide microRNAs for breast cancer detection. Clin Chim Acta 413: 1058-1065, 2012.

24. Gu YQ, Gong G, Xu ZL, et al: miRNA profiling reveals a potential role of milk stasis in breast carcinogenesis. Int J Mol Med 33: 1243-1249, 2014.

25. Dontula R, Dinasarapu A, Chetty C, et al: MicroRNA 203 modulates glioma cell migration via Robo1/ERK/MMP-9 signaling. Genes Cancer 4: 285-296, 2013.

26. Alajez NM, Lenarduzzi M, Ito E, et al: MiR-218 suppresses nasopharyngeal cancer progression through downregulation of survivin and the SLIT2-ROBO1 pathway. Cancer Res 71: 2381-2391, 2011

27. Khusial PR, Vadla B, Krishnan H, et al: Src activates Abl to augment Robol expression in order to promote tumor cell migration. Oncotarget 1: 198-209, 2010.

28. Yang L, Li Q, Wang Q, Jiang Z and Zhang L: Silencing of miRNA-218 promotes migration and invasion of breast cancer via Slit2-Robol pathway. Biomed Pharmacother 66: 535-540, 2012.

29. Tie J, Pan Y, Zhao L, et al: MiR-218 inhibits invasion and metastasis of gastric cancer by targeting the Robol receptor. PLoS Genet 6: e1000879, 2010. 